\title{
ON THE STUDY OF TRUE TUMOURS OF THE OPTIC NERVE.
}

BT PROFESSOR A. PICK, OF PRAGUE.

The last few years have seen the appearance in rapid succession not only of numerous isolated contributions but also of large comprehensive works on true tumours of the optic nerves. In face of what thus appears to be the defined position of teaching with respect to this condition, fresh observations have to prove their right to publication; the opinion that this can be allowed to a subsequently recorded case by the fact that from various clinical and pathological points of view it may properly be regarded as unique, induces me to publish the following one as shortly as possible.

On March 13, 1891, Victoria R., the wife of a labourer, was transferred from Professor Sattler's eye clinic to that for psychiatry, as she appeared to be abnormal mentally. The history of the case from the eye clinic was as follows:-

The patient, admitted there on November 10,1900, stated that about five weeks before she had sustained an injury to the left temporal region by striking violently against a broad wooden peg in a stable. Before the injury she had always been able to see well, and the evening before had even threaded a sewing needle by moon-light ; immediately after the injury a fluid began to escape in drops from the left nostril and continued to so for about a week. Before this the patient had never been ill and had given birth to two children; since the last confinement two years ago the menses had ceased.

The general bodily examination which, considering the state of the eyes, was conducted with especial regard to the nervous system, and also by the writer, showed nothing abnormal; uterine examination showed post-partum suprainvolution of the uterus; there was also constipation. 
The report of the examination of the eyes runs as follows: Externally, no signs of inflammation, eye movements follow promptly as ordered in all directions, the left eye shows slight permanent divergence, pupils both dilated and fixed, the media clear. Right papilla hyperæmic, swollen; the swelling measures about two to three dioptres and spreads some way on to the retina; the hyperæmia is an arterial one, showing a large number of fine vessels on the papilla; the veins also are distended and show a very tortuous course, there are no changes in the retina. Left papilla greatly swollen, the swelling being unequally distributed, the upper half and a three-cornered prism of the lower half being prominent. The hyperæmia on this side is also mainly arterial, a network of the finest vessels being spread over the papilla, the veins are also greatly distended and tortuous, the swelling is limited to the papilla, so that it stands out steeply from the surrounding retina which is normal, except for some changes mentioned below. These changes consist of small yellow dots which lie in the retina, are found to be under the vessels and are scattered over the whole field of the retina, but especially outwards and downwards from the papilla arranged in a line like a rosary.

Examination of functions. - The patient's statements are quite untrustworthy; so much can only be ascertained with certainty: that the vision of the left eye is better than the right, and colours are more quickly recognised ; after repeated attempts the patient occasionally recognised small objects such as the hand of a watch; the light sense could not be tested because the patient stated she saw absolutely nothing in the dark.

Rhinoscopic and aural examination revealed nothing more than slight swelling of the mucous membrane of the left nasal cavity.

From the case book of the eye clinic the following extracts are given :-

November 20.-Papillæ on both sides greatly swollen, the left more than the right, on the left side the dotting (stippling) is more evident, the right papilla is already dirty gray. Blue and yellow recognised with certainty, but patient has red-green blindness.

December 8.-Diminution of swelling of right papilla, with undoubted transition into atrophy; upper half of left papilla still inuch swollen, but not the lower.

December 16. - Right, no essential alteration ; left, the swelling less, the lower half of the papilla is changing to a dirty colour. 
December 27.--Patient has wandered at night, says the Virgin Mary has appeared to her and has ordered her poultices to the abdomen.

January 14.-Left papilla pale ; right, the upper half swollen, only a thick net-work of dilated vessels being visible there, the stipplings are visible scattered over the whole of the retina. Red-green blindness, blue and yellow well recognised, field of vision only examined with the hand, but appears from very untrustworthy answers not to be contracted.

February 1.-On both sides marked increase of atrophy; left papilla pale with a tinge of dirty yellow, vessels already narrowed; right, only above is swelling to be seen, the vessels still of normal size.

February 18.-Restless at night ; says God has appeared to her. Complains of paræsthesia in the left leg and nape of the neck, disappearing after a laxative.

March 1.-Pronounced atrophy of both optic nerves, the transition from papillitis still visible in the left.

On March 13 the patient was, as already mentioned, transferred to the clinic for psychiatry as she was more frequently ballucinated and restless. There she was in the first few days scarcely approachable, prayed almost constantly, would eat nothing, reacted violently against any touch; in calm intervals protested against her transfer, at night was still very restless.

On March 17 it was at last possible to make a thorough examination. She gave correct information considering her general condition and history; her recent conduct she explained chiefly by hallucinations. The fresh examination, especially of the nervous system, revealed nothing abnormal except the optic nerve atrophy. Subsequently the patient was often restless, occasionally violent to other patients because they made a " noise and bad smell"; at times quiet, asking to be discharged, and saying if it is God's will she will soon be well; she asked to see an advocate in order to arrange some business.

March 24.-Ophthalmoscopic examination.-Papillæ white, arteries narrow. Can see fingers at one metre; complains of headache. It is noteworthy that at repeated examinations the pupils now reacted both directly and consensually, the right more quickly.

March 26.-At 10.30 p.m., she suddenly cried out, breathed noisily, became cyanotic and had shaking movements of the arms, after which she slept on into the next day. The attack was not observed by a medical man.

March 27.-Complaint of beadache, mark of biting on right 
edge of tongue, the left upper extremity perhaps not so steady as the right when held out, face equal, tendency to foot clonus, the left slightly more so, pupils not acting to light. Enema, ice-bag. In the afternoon about seventeen epileptic seizures of varying intensity, passing one into another in quick succession; after these, greatly increased knee-jerk and brisk foot clonus.

March 28. - In spite of repeated enemata no action of the bowels. Patient stuporous. Chloral hydrate given per rectum, in consequence of further recurrence of status epilepticus, in which the patient died on March 30 , the temperature having risen steadily to $39^{\circ} \mathrm{C}$.

The autopsy performed the next day in Professor Chiari's anatomico-pathological institute gave the following results:-

Edema of the brain, gray degeneration of the optic nerves, bilateral lobular pneumonia, bronchitis, obsolete tubercle at the pulmonary apices and in the peri-bronchial glands. The following passage from the report is noteworthy. The optic nerves markedly gray in colour although not diminished in size. If this was remarkable the microscopic examination was equally so. In the first place, examination of Weigert-Pal preparations showed that both optic nerves, the chiasma, and also the stump of the optic tract did not exhibit typical staining, so that an inexperienced observer would have made a diagnosis of atrophy of the optic nerves. A closer examination, however, showed that even with this stain and without regard to the incongruity already observed macroscopically between evident atrophy and absence of any wasting, there were found in the transverse section, beside numerous round cells, peculiarly shaped bodies of varying size furnished with long processes, and that moreover, the sheath of the optic nerve was considerably thickened. In spite of some difficulty which arose from the fact that the whole specimen had already been hardened for a long time in Müller's fluid it was attempted to make a teased specimen, which sufficed to show that in addition to the completely atrophied nerve fibres and a moderate thickening of the vessels there were on the one hand masses of round cells and on the other the typical or angular cells of myxoma with often extraordinarily long

vol. XXIV. 
spirally twisted processes, thus marking the tumour as a myxosarcoma.

The special points of this case are so evident that they only need a short summary.

Before all, stands the question of diagnosis; tumour of the optic nerves was not diagnosed. A latent affection of brain in connection with the suprainvolution of the uterus was much more considered, indeed, the absence of a diagnosis still seems justified, Braunschweig, who recently collected cases of genuine tumour of the optic nerve (Graefe's Archiv. Bd. 39, Abth. 4, p. 24), having found that exophthalmos is never absent in such cases; our present case is, however, appropriate to this point, and shows the importance of the little word "never," already discarded in medicine, being limited in its applicability.

The present case should also cause the early development of visual defect to be regarded as of diagnostic importance, inasmuch as the contrast to the condition in choked disc the result of cerebral disease compels us to lay important stress on the direct disturbance of the optic nerves caused by the infiltrating tumour. Our case emphasises and confirms the statement of Braunschweig (loc. cit., p. 36) : "If characteristic ophthalmoscopic appearances for optic nerve tumours are not found, still the regular early occurrence of inflammation and swelling of the optic disc is conspicuous, and where palpation does not give evidence of a tumour and exophthalmos has not developed, a tumour of the optic nerves should occasionally be borne in mind, and at least a guarded prognosis should be given."

Noteworthy also is the etiological impetus. Considering the absence of any characteristic symptoms of tumour of the optic nerve, one was inclined in the present case to regard the trauma as the result of an already present but unobserved defect of vision; in this direction also the case may be instructive in that trauma is so often given as the cause of tumour of the optic nerve. Important also is the rapid course of our case in contrast to those previously described. Whether the slight strabismus observed, as in other similar cases, was due to the tumour must remain 
undecided in the absence of a reliable history. Further, the very remarkable facts with reference to the variable condition of the reflex pupillary reactions must be referred to. From the importance of this symptom one is reminded that similar symptoms in cerebral syphilis depend on the state of syphilitic granulations due to changes in nutrition resulting from vascular fullness; and in so far as in that condition the groundwork of the tumour growing in the optic nerves consists of a soft tissue rich in cells, we are perhaps, not wrong in giving a similar explanation here. To further careful observations must be left the decision as to how far this symptom may be of diagnostic value. Finally, stress must be laid on the fact that perhaps initial palpation of the optic nerves is of diagnostic value as inspection of the same would never have led to a diagnosis. We are therefore brought to the pathological anatorny of our case, which certainly is of no small interest. Firstly the position and extent of the tumour appears to me important, for as one cannot suppose that both optic nerves were separately and simultaneously diseased we are driven to the conclusion that apparently the chiasma was the original seat of the growth and that thence the spread took place on the one hand into both nerves and on the other into the tracts; secondly, the absence of any tumour bulging out the optic sheath from within appears to me to be important considering its longitudinal extent; further the absence of any otber abnormality in the shape of the optic nerves which so often in similar cases are bent like a post-horn. One can only suppose that the thickened sheath opposed a considerable resistance to the transverse growth of the mass of the tumour, and therefore led to a marked development in the long axis and so to speak to a purely infiltrating growth. Perhaps the unusually long extent of the tumour through the optic nerves and chiasma is thus to be explained.

Lastly, a few words as to the symptoms indicating the participation of the brain, which I think may be partly explained by what was found. First as to the psychosis which apparently was based essentially on hallucinations of sight. These corresponded undoubtedly with what one 
designates pure hallucinations of sight as they are seen in disease of the retina or optic nerves, this is confirmed by the subsequent occurrence also of hallucinations of hearing and smell. If the disease of the optic nerves was related to the first of which indeed it appears to have been the basis, it is perhaps not impossible that pressure of the tumour on the nerve trunks was partly responsible for both the latter. As to the meaning of the epileptic seizures, it must be remembered that in a perfectly unique case of Alt's (quoted by Braunschweig, loc. cit., p. 43), epileptic attacks were also observed as the case developed; Alt explains their occurrence by pressure on the parts of the central nervous system lying adjacent to the chiasma, but it appears to me that the facts point rather to the influence of a disturbance of the state of the circulation in the dural sheath of the optic nerves compressed from within outwards. 